\title{
CORRESPONDENCE
}

\section{Chronic myelomonocytic leukemia patients with RAS pathway mutations show high in vitro myeloid colony formation in the absence of exogenous growth factors}

Leukemia (2016) 30, 2280-2281; doi:10.1038/leu.2016.235; published online 2 September 2016

We read with great interest the article by Akutagawa et al. ${ }^{1}$ in this journal in which the authors describe that PI3K inhibitors unexpectedly profoundly inhibited myeloproliferation in an myelodysplastic/myeloproliferative neoplasm mouse model driven by hyperactive RAS, suggesting a new therapeutic strategy in juvenile myelomonocytic leukemia (JMML) and chronic myelomonocytic leukemia (CMML).

The basis for all RAS pathway-oriented treatment concepts is the identification of RAS pathway hyperactivation in patients. Due to the fact that in CMML more than one molecular aberration can be detected in the majority of patients, functional tests may be important to better estimate the contribution of a particular molecular aberration in the pathogenesis of the malignancy. ${ }^{2}$ In $J M M L$, in which molecular aberrations are mainly restricted to the RASopathy genes, including NRAS, KRAS, NF1, CBL and PTPN11, the spontaneous formation of colony-forming unit-granulocytemacrophage (CFU-GM) due to granulocyte/macrophage colonystimulating factor (GM-CSF)-specific hypersensitivity is a hallmark feature of disease, which has been included in the diagnostic criteria. $^{3}$

We have originally reported that extensive in vitro formation of CFU-GM without exogenous growth factors can also be found in a subset of patients with CMML. ${ }^{4}$ We demonstrated that this spontaneous myeloid colony formation in CMML is a GM-CSFdependent in vitro phenomenon and could also show that CMML patients with high spontaneous CFU-GM growth (>100/10 5 PBMNC) have a worse prognosis compared with patients with low CFU-GM growth, suggesting a clinical significance of our observation.,

We speculated that spontaneous myeloid colony formation might be a surrogate parameter of RAS pathway hyperactivation in CMML. To test this hypothesis, we performed next-generation sequencing (NGS) from stored peripheral blood mononuclear cells (PB MNC) obtained from $100 \mathrm{CMML}$ patients, in whom in vitro cultures have been performed during the last years. In vitro culture data were then correlated with molecular aberrations of RAS pathway components.

The diagnosis of CMML was made according to the diagnostic criteria of the World Health Organization classification of $2008 .^{7}$ For molecular characterization, we used NGS with amplicon-based target enrichment of $39 \mathrm{CMML}$-associated genes, including $A S X L 1$, EGFR, KRAS, SF3A1, ATRX, ETV6, MET, SF3B1, BCOR, EZH2, NF1, SRSF2, BRAF, FLT3, NPM1, STAG2, CBL, GNAS, NRAS, TET2, CDKN2A, IDH1, PRPF40B, TP53, CEBPA, IDH2, PTPN11, U2AF1, CSF1R, JAK2, RUNX1, WT1, CSF3R, KDM6A, SETBP1, ZRSR2, DNMT3A, KIT and SF1. Assuming that clones that are too small are unlikely to significantly impact hematopoiesis, only mutations with an allele burden of $20 \%$ or higher were considered as positive for analysis. CFU-GM growth in the absence of exogenous cytokines was assessed using semisolid cultures as previously described. ${ }^{5}$

In $40 \mathrm{CMML}$ patients, mutations in at least one of the RASopathy genes were detected; in 60 patients, no mutations in RAS pathway components or such mutations with allele frequencies $<20 \%$ were found. In the 40 patients with RAS pathway mutations, we found molecular aberrations of the NRAS gene in 19, KRAS in 6, NF1 in 3, CBL in 10 and PTPN11 in 2 patients, respectively. Mutations of RAS pathway components were mutually exclusive, only low levels of more than one RASopathy mutations were found in some patients. In all patients with RAS pathway mutations, additional mutations were observed in other genes, particularly in components of DNA methylation and/or the spliceosome as previously reported by others. ${ }^{2}$ Results of semisolid cultures show that in CMML patients in whom molecular aberration in RAS pathway components could be detected had a much higher spontaneous myeloid colony formation than CMML patients without RAS pathway mutations (Figure 1). The median number of spontaneously formed CFU-GM $/ 10^{5}$ MNC was 147.5 (range 0-1009) in RAS-positive patients as compared with $2(0-812)$ in RAS-negative patients $(P<0.00001$ by the Wilcoxon's rank-sum test). Unstimulated myeloid colony formation in RAS-positive CMML patients is also much higher than the spontaneous formation of CFU-GM in normal individuals (median 4.8/10 $0^{5}$ PBNC, range 3.5-8.5), which has been reported by us previously. ${ }^{8}$ The incidence of RAS pathway mutations was $72 \%(21 / 29)$ in CMML patients with

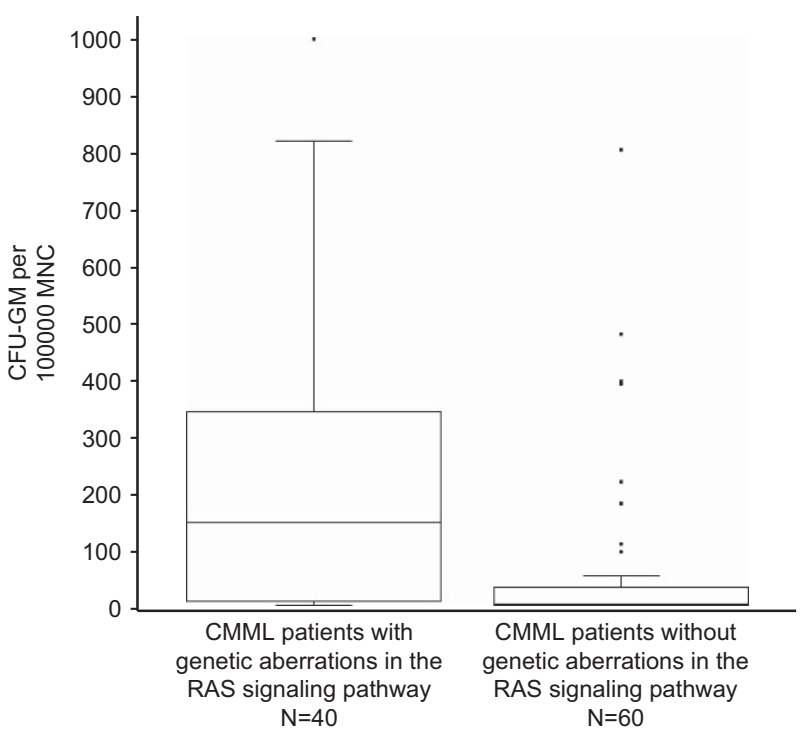

Figure 1. Comparison of spontaneous in vitro CFU-GM growth from PBMNC in CMML patients with and without mutations in RASopathy genes, including NRAS, KRAS, NF1, CBL and PTPN11. CFU-GM formation in the absence of exogenous cytokines was assessed using semisolid cultures as previously described. ${ }^{5}$ Colony numbers are shown as box plots with first and third quartiles and interquartile ranges. Data were analyzed using the Wilcoxon's rank-sum test. Spontaneous myeloid colony formation was significantly higher in CMML patients with mutations in RAS pathway components than in patients without such mutations $(P<0.00001)$. 
high-colony growth ( $>100 / 10^{5}$ PBMNC) and $27 \%(19 / 71)$ in patients with low spontaneous CFU-GM formation $(P<0.0001$ by the chi square test). In eight patients, high CFU-GM growth was observed without evidence of genetic aberrations in RAS signaling. This may indicate that additional molecular aberrations of the RAS pathway, which are not covered by our targeted NGS panel, may cause spontaneous cell proliferation, or, alternatively, that other signaling pathways may also play a certain role in this in vitro phenomenon.

Our findings suggest that high spontaneous myeloid colony growth in CMML is significantly associated with molecular aberrations of genes involved in RAS signaling and thus seems to reflect RAS pathway hyperactivation in patients with CMML. Therefore, spontaneous colony formation in semisolid cultures could be a helpful functional test to identify patients who are potential candidates for treatment concepts designed for patients with RAS pathway-driven hematologic malignancies.

\section{CONFLICT OF INTEREST}

The authors declare no conflict of interest.

\section{K Geissler ${ }^{1}$, E Jäger ${ }^{2}$, A Barna ${ }^{3}$, T Alendar ${ }^{1}$, E Ljubuncic $^{1}$, T Sliwa ${ }^{4}$ and $\mathrm{P}$ Valent ${ }^{5}$}

${ }^{1}$ Fifth Medical Department with Hematology, Oncology and Palliative Medicine, Hospital Hietzing, Vienna, Austria;

${ }^{2}$ Department of Laboratory Medicine, Medical University of Vienna, Vienna, Austria;

${ }^{3}$ Blood Transfusion Service for Upper Austria, Austrian Red Cross, Linz, Austria;

${ }^{4}$ Third Medical Department, Hanusch Hospital, Vienna, Austria and

${ }^{5}$ Division of Hematology and Hemostaseology, Department of Internal Medicine I, Medical University of Vienna, Vienna, Austria E-mail: klaus.geissler@wienkav.at

\section{REFERENCES}

1 Akutagawa J, Huang TQ, Epstein I, Chang T, Quirindongo-Crespo M, Cottonham CL et al. Targeting the PI3K/Akt pathway in murine MDS/MPN driven by hyperactive RAS. Leukemia 2016; 30: 1335-1343.

2 Itzykson R, Kosmider O, Renneville A, Morabito M, Preudhomme C, Berthon C et al. Clonal architecture of chronic myelomonocytic leukemias. Blood 2013; 121: 2186-2198.

3 Niemeyer CM. RAS diseases in children. Haematologica 2014; 99: 1653-1662.

4 Geissler K, Hinterberger W, Bettelheim P, Haas O, Lechner K. Colony growth characteristics in chronic myelomonocytic leukemia. Leuk Res 1988; 12: 373-377.

5 Geissler K, Ohler L, Födinger M, Virgolini I, Leimer M, Kabrna E et al. Interleukin 10 inhibits growth and granulocyte/macrophage colony-stimulating factor production in chronic myelomonocytic leukemia cells. J Exp Med 1996; 184: 1377-1384.

6 Sagaster V, Ohler L, Berer A, Kabrna E, Ofner P, Lechner K et al. High spontaneous colony growth in chronic myelomonocytic leukemia correlates with increased disease activity and is a novel prognostic factor for predicting short survival. Ann Hematol 2004; 83: 9-13.

7 Vardiman JW, Thiele J, Arber DA, Brunning RD, Borowitz MJ, Porwit A et al. The 2008 revision of the World Health Organization (WHO) classification of myeloid neoplasms and acute leukemia: rationale and important changes. Blood 2009; 114: 937-951.

8 Oehler L, Foedinger M, Koeller M, Kollars M, Reiter E, Bohle B et al. Interleukin-10 inhibits spontaneous colony-forming unit-granulocyte-macrophage growth from human peripheral blood mononuclear cells by suppression of endogenous granulocyte-macrophage colony-stimulating factor release. Blood 1997; 89: $1147-1153$.

(c) (i) $\odot$ This work is licensed under a Creative Commons Attributioncc) NonCommercial-NoDerivs 4.0 International License. The images or other third party material in this article are included in the article's Creative Commons license, unless indicated otherwise in the credit line; if the material is not included under the Creative Commons license, users will need to obtain permission from the license holder to reproduce the material. To view a copy of this license, visit http:// creativecommons.org/licenses/by-nc-nd/4.0/

(c) The Author(s) 2016 